\title{
Entropy of Leukemia on Multidimensional Morphological and Molecular Landscapes
}

\author{
Jose M. G. Vilar \\ Biophysics Unit (CSIC-UPV/EHU) and Department of Biochemistry and Molecular Biology, \\ University of the Basque Country, P.O. Box 644, 48080 Bilbao, Spain \\ and IKERBASQUE, Basque Foundation for Science, 48011 Bilbao, Spain \\ (Received 3 February 2014; published 28 May 2014)
}

\begin{abstract}
Leukemia epitomizes the class of highly complex diseases that new technologies aim to tackle by using large sets of single-cell-level information. Achieving such a goal depends critically not only on experimental techniques but also on approaches to interpret the data. A most pressing issue is to identify the salient quantitative features of the disease from the resulting massive amounts of information. Here, I show that the entropies of cell-population distributions on specific multidimensional molecular and morphological landscapes provide a set of measures for the precise characterization of normal and pathological states, such as those corresponding to healthy individuals and acute myeloid leukemia (AML) patients. I provide a systematic procedure to identify the specific landscapes and illustrate how, applied to cell samples from peripheral blood and bone marrow aspirates, this characterization accurately diagnoses AML from just flow cytometry data. The methodology can generally be applied to other types of cell populations and establishes a straightforward link between the traditional statistical thermodynamics methodology and biomedical applications.
\end{abstract}

DOI: 10.1103/PhysRevX.4.021038

\section{INTRODUCTION}

Many complex diseases require the measurement of multiple molecular factors at the single-cell level over large populations of cells for their precise characterization and detailed understanding [1-3]. Current technologies, such as flow cytometry (FCM), allow for such measurements, including simultaneous quantification of several morphological and molecular properties [4,5]. Currently, these technologies can provide the simultaneous single-cell measurement of tens of surface and intracellular markers of up to thousands of cells per second [6,7]. Such rapid technological development, however, is yet to be matched by traditional analysis tools to interpret the data $[8,9]$. It is now clear that all of these data by themselves, without the analytical tools to extract the relevant information, are not enough to faithfully understand the underlying cellular processes and their dysregulation in diseases such as cancer.

A prototypical example in which large amounts of data are generated is the cytometric analysis of acute myeloid leukemia (AML), a type of cancer produced by the dysregulated growth of the myeloid line of blood cells [10]. AML leads to abnormal white blood cells, red blood cells, or platelets accumulating in the bone marrow and

Published by the American Physical Society under the terms of the Creative Commons Attribution 3.0 License. Further distribution of this work must maintain attribution to the author(s) and the published article's title, journal citation, and DOI.
Subject Areas: Biological Physics, Complex Systems, Interdisciplinary Physics

blood, which interferes with the production of normal blood cells. The presence of abnormal cells can potentially be detected by analyzing the changes of the distribution of morphological and molecular attributes of cell samples from blood or bone marrow. There are important difficulties associated with this approach for diagnosing AML. In addition to abnormal cells being mixed with normal cells in the population samples, there are several AML subtypes and there is not an obvious well-defined set of changes in the molecular attributes that can fully characterize AML. In addition, there is the natural variation between individuals that overlaps with the changes induced by AML. Thus, the main challenge is to identify the quantities that can best be used to distinguish between AML patients and normal (AML-free) individuals based on the single-cell statistical properties of their blood or bone marrow cell populations.

Here, I show that entropy, as traditionally used in statistical thermodynamics [11], provides a measure for the precise diagnosis of AML. Cell populations are characterized by their entropies in multidimensional landscapes constructed from the distributions of single-cell morphological and molecular attributes of flow cytometry data. Diagnosis is achieved by comparing how far the cell population distribution of an individual is from the AML and normal prototypical maximum-entropy distributions.

The approach presented here is applied to samples from peripheral blood and bone marrow of 359 patients provided at the DREAM6/FlowCAP2 Molecular Classification of AML Challenge $[9,12]$. The results accurately diagnosed 
AML in a blind test set of 179 patients from just the provided training set of 180 patients, and ranked first among the best-performing methods of this challenge $[9,13]$. Challenges such as those put forth by DREAM (Dialogue for Reverse Engineering Assessments and Methods) and FlowCAP (Flow Cytometry: Critical Assessment of Population Identification Methods) provide objective unbiased evaluations of computational methods in complex situations and eliminate the possibility of overfitting since the results are not known in advance $[9,14-16]$. The different approaches that participated at the DREAM6/FlowCAP2 challenge included mostly traditional machine learning algorithms, such as support vector machine regression, logistic regression, vector quantization, and correlative matrix mapping. Overall, most of the approaches obtained good results [9].

\section{GENERAL APPROACH}

Leukemia, as any other type of cancer, results from dysregulated growth caused by genetic and epigenetic changes that alter the cellular state [17]. Therefore, the first step towards distinguishing cancerous from normal cell populations is to characterize the state of each cell. To take into account that there is only limited information, the approach considers measured $x$ and internal (nonmeasured) $q$ quantities separately. In the case of FCM, light scattering and the intensity of fluorescent reporters are the prototypical examples of measured quantities. Internal quantities are much more numerous and include, among others, nonmeasured protein levels and specific DNA mutations. The characterization of a population $i$ considers the probability distribution $P_{i}(x, q)$ of these two types of attributes among its cells.

The goal is to discriminate among different cellpopulation types based on the statistical properties of their measurable quantities. To be able to take into account the effects of the internal quantities $q$, one must estimate their effects from the measurable quantities $x$, which should carry sufficient information about the key cellular differences between the different population types.

A convenient starting point to estimate the effects of the internal quantities in a cell population is the entropy of its attributes' distribution $S_{i}=-\int P_{i}(x, q) \ln P_{i}(x, q) d x d q$. This quantity can be expressed as a function of just $x$ by rewriting the joint probability $P_{i}(x, q)=P_{i}(q \mid x) P_{i}(x)$ in terms of the conditional probability of $q$ with respect to $x$, $P_{i}(q \mid x)$, and the probability of $x, P_{i}(x)$. Integration over $q$ leads to

$$
S_{i}=-\int P_{i}(x)\left[f_{i}(x)+\ln P_{i}(x)\right] d x,
$$

which explicitly encapsulates the contributions from internal quantities into the function $f_{i}(x)=$ $\int P_{i}(q \mid x) \ln P_{i}(q \mid x) d q$.
The main hypothesis needed to proceed further is that the internal contribution for each population $f_{i}(x)$ can accurately be approximated by the same function $f_{T}(x)$ for all of the members of a given type $T$. In the case of AML and normal types, the result would be $f_{i}(x)=f_{\mathrm{AML}}(x)$ or $f_{i}(x)=f_{\text {normal }}(x)$, depending on whether the population $i$ is AML or normal.

The function $f_{T}(x)$ can be estimated by considering a reference maximum-entropy distribution $P_{T}(x)$ that integrates the main features of the cell populations of type $T$. The maximum condition implies that $\delta S_{T}=$ $-\int \delta P_{T}(x)\left[f_{T}(x)+\ln P_{T}(x)\right] d x$ is zero, which happens when the term multiplying the variation of the probability in the integrand is constant. This condition can be rewritten as $f_{T}(x)=-\ln P_{T}(x)-S_{T}$, where the constant $S_{T}$ is the maximum entropy for the cell population type $T$. Therefore, using $f_{i}(x)=f_{T}(x)=-\ln P_{T}(x)-S_{T} \quad$ in Eq. (1) leads to

$$
S_{i, T}=S_{T}-\int P_{i}(x) \ln \left[P_{i}(x) / P_{T}(x)\right] d x,
$$

which can be interpreted as the entropy of the population $i$ on the multidimensional morphological and molecular landscape defined by the maximum-entropy distribution for the cell population type $T$.

The explicit reference distribution $P_{T}(x)$ is chosen as the distribution that maximizes the total entropy of all the members of type $T$. The variation of the total entropy with respect to $P_{T}(x)$ is given by $\delta\left(\sum_{i \in T} S_{i, T}\right)=$ $-\int \delta P_{T}(x)\left[\sum_{i \in T} P_{i}(x) / P_{T}(x)\right] d x$, which is zero when $P_{T}(x)$ is proportional to $\sum_{i \in T} P_{i}(x)$. Therefore, $P_{T}(x)$ is chosen to be the average distribution, which for AML and normal types is given by $P_{\mathrm{AML}}(x)=$ $\left(1 / N_{\mathrm{AML}}\right) \sum_{i \in \mathrm{AML}} P_{i}(x)$ and $P_{\text {normal }}(x)=\left(1 / N_{\text {normal }}\right)$ $\sum_{i \in \text { normal }} P_{i}(x)$, respectively, where $N_{\mathrm{AML}}$ and $N_{\text {normal }}$ are the number of cell populations for each type.

The explicit form of Eq. (2) shares many similarities with other expressions used in physical sciences and information theory. It has exactly the same form as the Gibbs entropy formula $[11,18,19]$. The main difference is that, in that case, the state $T$ refers to an equilibrium state instead of a cell population type. It is also very similar, except for the constant $S_{T}$, to the Kullback-Leibler divergence, which uses relative entropies to compare distributions [20]. It is important to emphasize that entropy defined by Eq. (2) does not quantify variability. Instead, it quantifies how similar the distribution of measured values for a given individual is to the expected average distribution for a given type. High entropy means that the distribution of the individual is similar to the expected distribution and low entropy means that it is different. Both high and low variability would lead to low relative entropy values if the underlying distributions were not close to the expected distribution.

A key feature of the method is that, since it is based on a maximum principle with respect to the distributions of the 
attributes, the effects of small perturbations in the measured distributions are second order and, therefore, they will only minimally impact the results.

The analogy with statistical thermodynamics can be extended further to estimate the likelihood $p_{i, T}$ of the population belonging to type $T$ through the Einstein fluctuation formula, $p_{i, T} \sim e^{S_{i, T}}$, which relates the probability of observing a state with its entropy [21,22]. After normalization, it results in

$$
p_{i, T}=\frac{e^{S_{i, T}}}{\sum_{T^{\prime}} e^{S_{i, T^{\prime}}}},
$$

where the sum in the denominator is performed over all of the cell population types. This expression assigns a high likelihood to a cell population as being of type $T$ if the distribution of its attributes has a form similar to the maximum-entropy distribution for that type. In many ways this assignment parallels the approach first used by Einstein to compute the probability of observing a fluctuation moving a system away from its equilibrium state based on its entropy change [22].

\section{APPLICATION TO DIAGNOSING LEUKEMIA}

The explicit application to diagnosing AML relies on evaluating whether the distribution of the values of flow cytometry data for a given individual to be diagnosed (Fig. 1; see also Fig. 1 in the Supplemental Material [23]) is closer to the AML or the normal maximum entropy distribution (Fig. 1). Using just a few variables, such as side scatter and a fluorescent marker for the receptor protein CD45, is informative in many cases but does not offer a full characterization (compare, for instance, AML patient no. 7 with normal patient no. 96 in Fig. 1 in the Supplemental Material [23]). In general, there are several sets of simultaneously measured variables for a given individual and, therefore, there are several different landscapes. For each population $i$ and each set $k$ of simultaneously measured variables $x^{k}$, the corresponding entropy is given by $S_{i, T}^{k}=S_{T}^{k}-\int P_{i}\left(x^{k}\right) \ln \left[P_{i}\left(x^{k}\right) / P_{T}\left(x^{k}\right)\right] d x^{k}$. For an AML individual, the values of the entropies are expected to be $S_{i, \mathrm{AML}}^{k}-S_{\mathrm{AML}}^{k} \approx 0$ and $S_{i, \text { normal }}^{k}-S_{\text {normal }}^{k} \ll 0$. A normal individual, in contrast, would lead to $S_{i, \mathrm{AML}}^{k}-$ $S_{\mathrm{AML}}^{k} \ll 0$ and $S_{i, \text { normal }}^{k}-S_{\text {normal }}^{k} \approx 0$. Therefore, the relative entropy difference,

$$
\begin{aligned}
\Delta S_{i}^{k}= & S_{i, \text { normal }}^{k}-S_{i, \mathrm{AML}}^{k} \\
= & S_{\text {normal }}^{k}-S_{\mathrm{AML}}^{k} \\
& -\int P_{i}\left(x^{k}\right) \ln \left[P_{\mathrm{AML}}\left(x^{k}\right) / P_{\text {normal }}\left(x^{k}\right)\right] d x^{k},
\end{aligned}
$$

indicates that the individual looks like an AML patient for negative values and like a normal subject for positive values. These entropies can be used to compute the weighted entropy difference
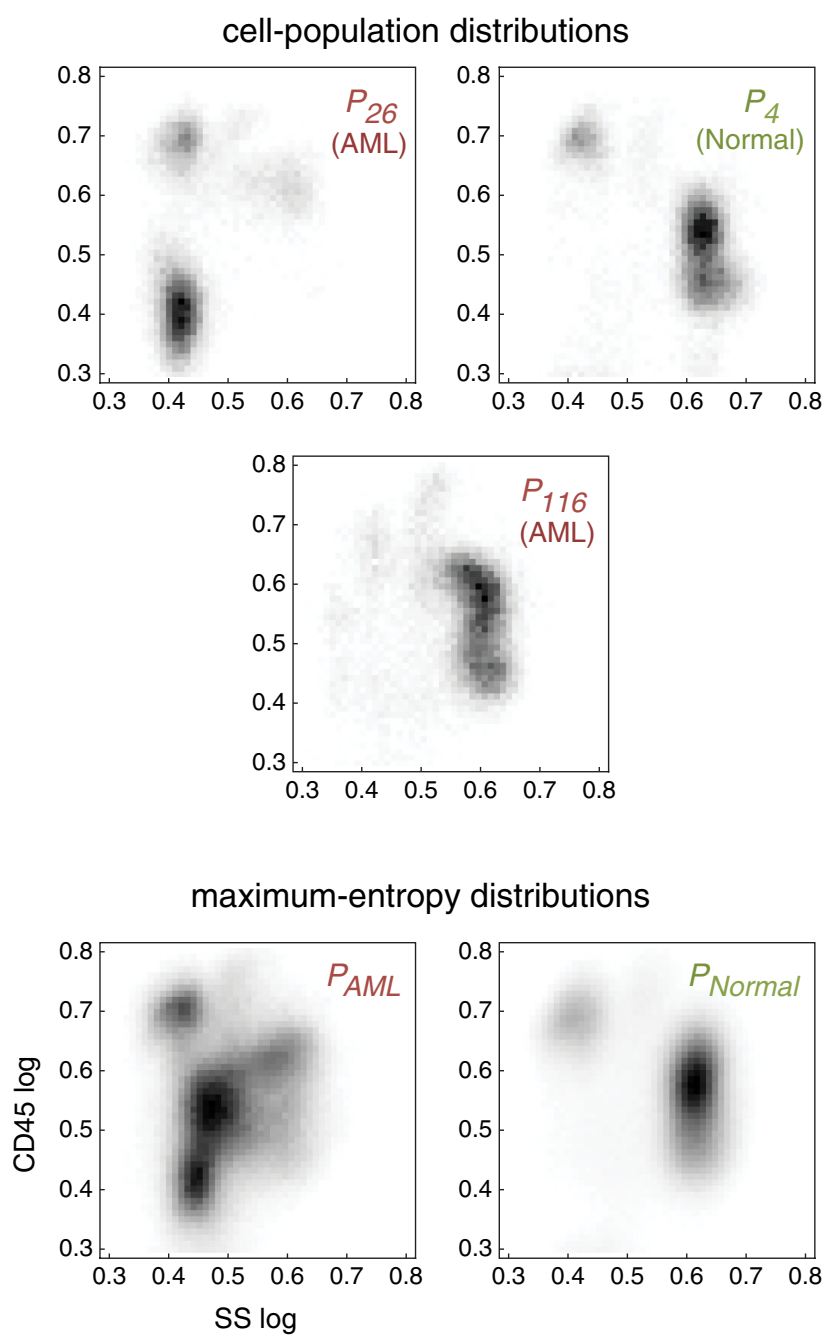

FIG. 1. Molecular and morphological landscapes of leukemia. The two-dimensional distribution of cells with given values of the logarithms of the side scatter (SS log) and the marker CD45 (CD45 log) intensities are shown for representative cell populations, with black and white representing high and zero densities, respectively. The distributions for AML patient no. $26\left(P_{26}\right)$ and normal individual no. $4\left(P_{4}\right)$ are indicative of distributions that closely resemble the maximum-entropy distribution of their state, either AML $\left(P_{\mathrm{AML}}\right)$ or normal $\left(P_{\text {normal }}\right)$ states. The distribution for AML patient no. $116\left(P_{116}\right)$, in contrast, does not show a clear discriminative feature of the presence of AML in the SS-CD45 landscape.

$$
\Delta \tilde{S}_{i}=\sum_{k} w_{k} \Delta S_{i}^{k}
$$

where $w_{k}$ is the weight of the set $k$. Similarly, the weighted entropies are defined by $\tilde{S}_{i, T}=\sum_{k} w_{k} S_{i, T}^{k}$ and $\tilde{S}_{T}=$ $\sum_{k} w_{k} S_{T}^{k}$. The likelihood for the population $i$ to be AML is estimated using Eq. (3) with the weighted entropy difference as

$$
p_{i, \mathrm{AML}}=\frac{1}{1+e^{\Delta \tilde{S}_{i}}} .
$$


TABLE I. List of the five specific fluorescent markers, denoted by FL1, FL2, FL3, FL4, and FL5, used in each of the seven groups of measurements.

\begin{tabular}{lccccc}
\hline \hline Group & FL1 & FL2 & FL3 & FL4 & FL5 \\
\hline 1 & IgG1-FITC & IgG1-PE & CD45-ECD & IgG1-PC5 & IgG1-PC7 \\
2 & Kappa-FIT & Lambda-PE & CD45-ECD & CD19-PC5 & CD20-PC7 \\
3 & CD7-FITC & CD4-PE & CD45-ECD & CD8-PC5 & CD2-PC7 \\
4 & CD15-FITC & CD13-PE & CD45-ECD & CD16-PC5 & CD56-PC7 \\
5 & CD14-FITC & CD11c-PE & CD45-ECD & CD64-PC5 & CD33-PC7 \\
6 & HLA-DR-FITC & CD117-PE & CD45-ECD & CD34-PC5 & CD38-PC7 \\
7 & CD5-FITC & CD19-PE & CD45-ECD & CD3-PC5 & CD10-PC7 \\
\hline \hline
\end{tabular}

\section{IMPLEMENTATION}

The DREAM6/FlowCAP2 data $[9,12]$ consists of seven groups of measurements for each individual, each group comprising the simultaneous measurements of the forward scatter (FS), the side scatter (SS), a fluorescent marker for the receptor protein CD45 (FL3), and a different combination of four different fluorescent markers for other proteins (FL1, FL2, FL4, and FL5). The specific fluorescent markers used as FL1, FL2, FL4, and FL5 in each group of measurements are listed in Table I. Therefore, there are seven different attribute spaces, described by the seven-dimensional vectors $x=(\mathrm{FS}, \mathrm{SS}, \mathrm{FL} 1, \mathrm{FL} 2, \mathrm{FL} 3$, FL4,FL5). For each space, the entropy can be computed in the full seven dimensions or in any combination of subspaces.

An avenue to estimate the suitability of a space or subspace for AML and normal individual discrimination is to use leave-one-out cross-validation with the training data. In this case, it consists of testing each individual $i$ of the training set without its contribution to $P_{\mathrm{AML}}$ or $P_{\text {normal }}$.

The results of cross-validation with uniform weights $w_{k}=1$ (Fig. 2; see also Fig. 2 in the Supplemental
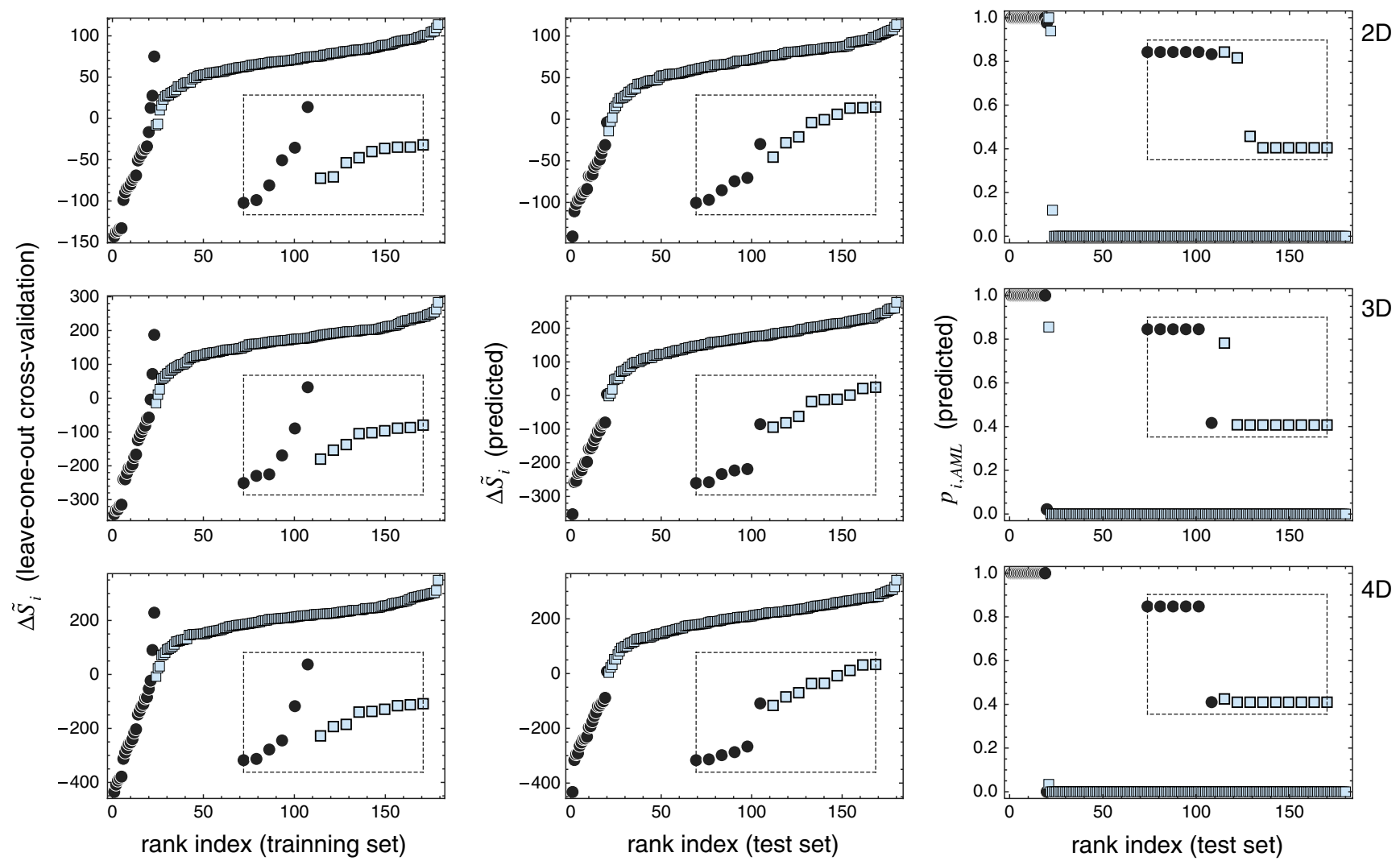

FIG. 2. Entropy-based discrimination between AML and normal individuals. The total relative entropy difference, using Eq. (5) with $w_{k}=1$, is shown for each individual of the training and test sets for two-, three-, and four-dimensional landscapes. The likelihood that an individual of the test set is AML positive, given by Eq. (6), is also shown for two-, three-, and four-dimensional landscapes. Circles and squares represent, respectively, the actual AML and normal state of a patient as clinically assessed by a physician. The insets are magnifications around the AML-normal boundary. 
Material [23]) indicate that normal and AML populations have high and low $\Delta \tilde{S}_{i}$ values, respectively, as expected from the assumptions of the approach. Only the population samples of just a few AML individuals have higher $\Delta \tilde{S}_{i}$ than the lowest $\Delta \tilde{S}_{i}$ for the normal set. Therefore, the assumption that the function $f_{i}(x)$ can be approximated by the maximum entropy distribution of its type holds for the cell populations of most individuals. In general, it is observed that for higher dimensionality spaces, the number of AML individuals that overlap with normal ones is smaller. Such a small overlap indicates that the entropy on multidimensional morphological and molecular landscapes provides an efficient avenue to discriminate AML from normal populations.

The results with the test set, using $P_{\mathrm{AML}}$ and $P_{\text {normal }}$ computed from the training set, show even better segregation of individuals based on the entropy of their cellpopulation samples (Fig. 2; see also Fig. 2 in the Supplemental Material [23]). It is possible to use Eq. (6) to estimate the likelihood of an individual being AML positive. However, the values $S_{\mathrm{AML}}$ and $S_{\text {normal }}$ for the maximum entropy of AML and normal individuals are not readily available. A first approximation, based on the crossvalidation results, is to consider $S_{\mathrm{AML}}-S_{\text {normal }} \approx 0$. The results indicate that the approach accurately diagnoses AML, except for just a few individuals (Fig. 2; see also Fig. 2 in the Supplemental Material [23]), and that the performance increases with the dimensionality of the space in which the entropy is computed. The results also suggest that the test data were easier to classify than the training data, which contains a few difficult patients.

The discriminative capabilities can be improved by selecting, among the many possibilities available, not only the dimensionality of the space but also the subspaces. The subspace can be chosen by selecting the weights $w_{k}$ to be either 0 or 1 . For instance, a combination that proved to give good results is the four-dimensional distributions for the values of $x^{k}=(\mathrm{FS}, \mathrm{SS}, \mathrm{FL} 3, k)$ with $k \in$ $\{$ FL1, FL2, FL4, FL5 $\}$ for the seven groups of measurements. Taking into account that the markers denoted as FL1, FL2, FL4, and FL5 are different in each group of measurements, there are $7 \times 4=28$ landscapes for each individual. The predictions obtained with this approach ranked first among the best performers of the DREAM6 competition (see the Supplemental Material [23]). Further improvement can be achieved by adjusting the values of the weights to maximize the segregation between AML and normal in the cross-validation phase.

A systematic procedure for adjusting the weights $w_{k}$ in Eq. (5) to better segregate between AML and normal individuals is to increase the weights that benefit the segregation and decrease the weights that worsen it. Segregation is quantified by the distance between the normal $N_{\min }$ and AML $A_{\max }$ individual with the minimum and maximum entropy difference, respectively. This distance is explicitly defined by $\Delta \Delta \tilde{S}=\sum_{k} w_{k} \Delta \Delta S^{k}$, with $\Delta \Delta S^{k}=\Delta S_{i=N_{\min }}^{k}-\Delta S_{i=A_{\max }}^{k}$. The procedure considers updates proportional to a small quantity $\Delta t$ so that the weights at the step index $t$ are updated iteratively to the step index $t+\Delta t$ through the expression

$$
w_{k}(t+\Delta t)=\frac{w_{k}(t)\left(1+\Delta t \Delta \Delta S^{k}\right)}{\sum_{k} w_{k}(t)\left(1+\Delta t \Delta \Delta S^{k}\right)}
$$

This procedure guarantees that $\Delta \Delta \tilde{S}$ increases in each interaction if $\Delta t$ is sufficiently small and the identities of

(a)
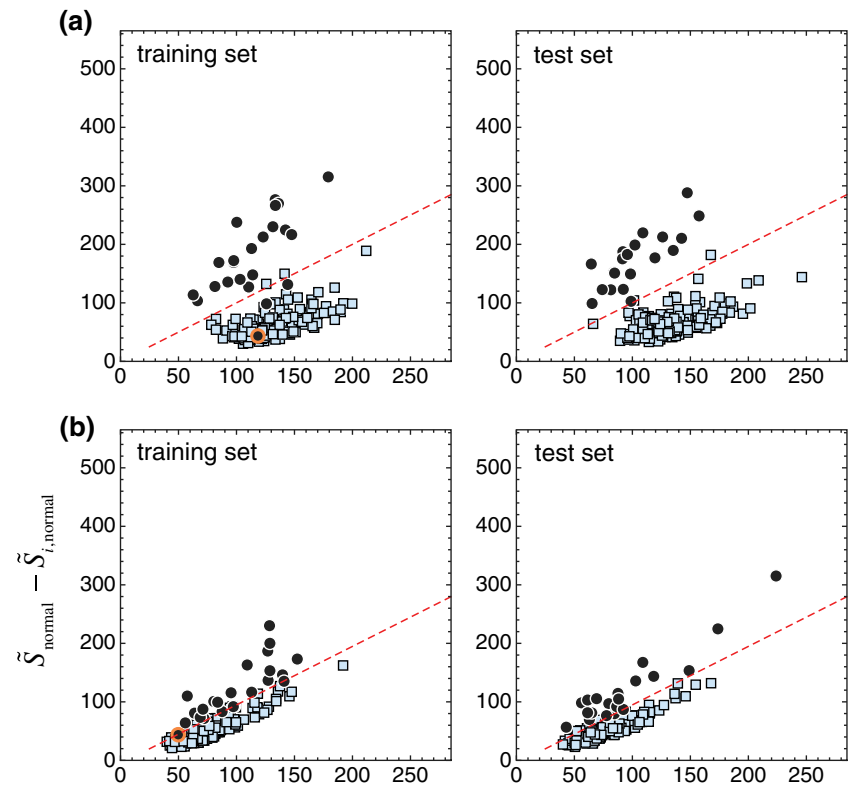

(c)

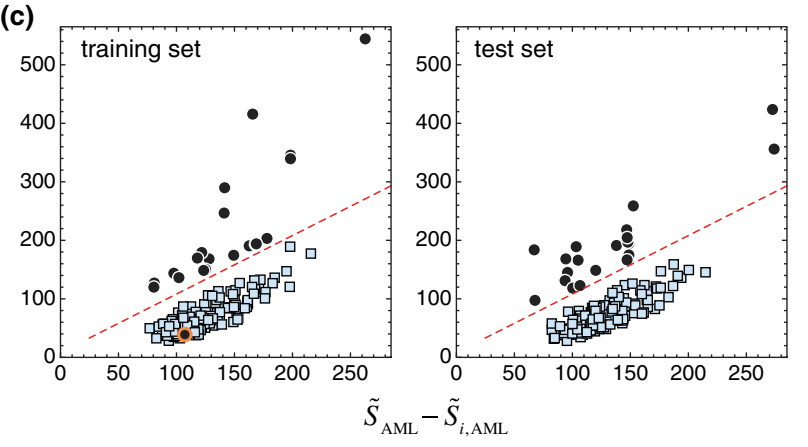

FIG. 3. Total and weighted entropies on the two-dimensional landscapes defined by the maximum-entropy distribution for AML and normal states. The entropies for the training (left-hand column) and test (right-hand column) sets were computed from Eq. (5) with uniform $w_{k}$ (a), with the $w_{k}$ that maximize the distance $\Delta \Delta \tilde{S}$ of the training set (b), and with the $w_{k}$ that maximize the distance $\Delta \Delta \tilde{S}$ of the training set excluding patient no. 116 (c). Circles and squares represent, respectively, the actual AML and normal state of a patient as clinically assessed by a physician. The dashed lines are given by $\tilde{S}_{\tilde{S} \text { normal }}-\tilde{S}_{i \text {,normal }}=$ $\tilde{S}_{\mathrm{AML}}-\tilde{S}_{i, \mathrm{AML}}+\Delta \Delta \tilde{S}\left(t_{\max }\right) / 2$ with the value of $\Delta \Delta \tilde{S}\left(t_{\max }\right) / 2$ equal to 0 (a), -5.21 (b), and 8.15 (c). The black circle with the orange contour corresponds to AML patient no. 116. 

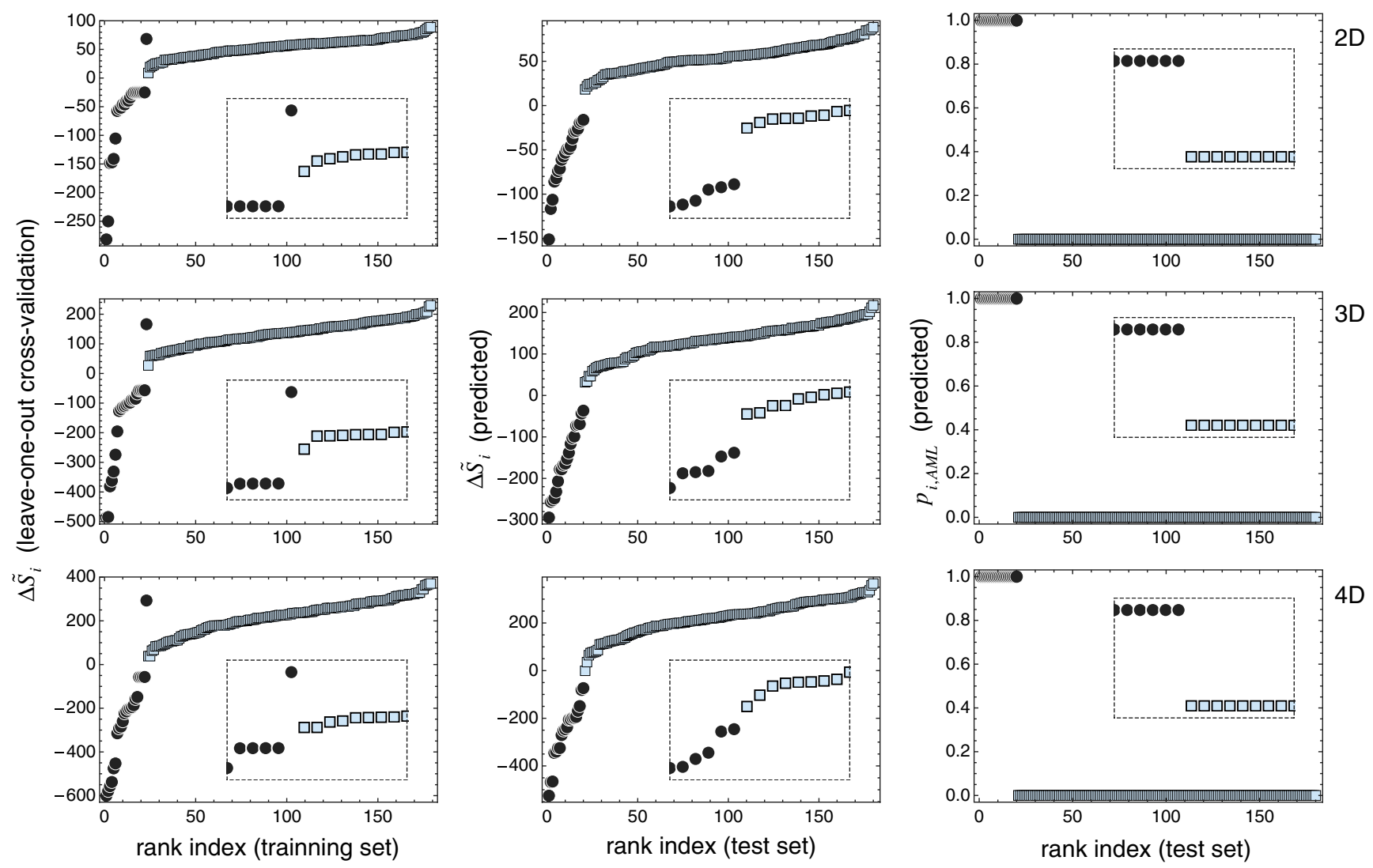

FIG. 4. Weighted-entropy enhancement of the discrimination between AML and normal individuals. Same situation as in Fig. 2 but with the weights $w_{k}$ that maximize the distance $\Delta \Delta \tilde{S}$ of the training set excluding patient no. 116.

the individuals $N_{\min }$ and $A_{\max }$ do not change. Under these assumptions, expanding Eq. (7) in $\Delta t$ and using $\Delta \Delta S(t)^{2}=$ $\sum_{k} \Delta \Delta S(t)^{2} w_{k}(t)$ leads to $\Delta \Delta \tilde{S}(t+\Delta t) \approx \Delta \Delta \tilde{S}(t)+$ $\Delta t \sum_{k}\left[\Delta \Delta S^{k}-\Delta \Delta S(t)\right]^{2} w_{k}(t)$, which shows that the increase is proportional to a combination of positive quantities.

The enhancement of the discrimination between AML and normal individuals by adjusting the weights $w_{k}(t)$ according to Eq. (7) is illustrated by the values of the entropies of each population $i$ with respect to AML, $\tilde{S}_{i, \mathrm{AML}}-\tilde{S}_{\mathrm{AML}}$, and normal, $\tilde{S}_{i, \text { normal }}-\tilde{S}_{\text {normal }}$, states. For $w_{k}=1$, AML and normal populations tend to be separated according to their entropies, but there is a significant overlap [Fig. 3(a)]. By updating the weights $w_{k}$ according to Eq. (7)), the quantity $\Delta \Delta \tilde{S}$, which is negative, is increased by decreasing its absolute value. This quantity never changes to positive values and there is not an actual segregation of the populations [Fig. 3(b)]. Closer inspection indicates that segregation is prevented by a patient in the AML training set (patient no. 116 in Fig. 1). By removing this patient from the calculation of $A_{\max }$, there is a clear segregation of AML and normal individuals according to the entropies of their distributions [Fig. 3(c)]. Perfect segregation occurs in both cross-validation with the training set, which was used to obtain the different parameters, and predictions with the test set, which is completely independent of the training set. As shown in Fig. 3(c), the reason patient no. 116 prevented segregation is because this patient has the normal instead of the typical AML signatures.

The segregation measure $\Delta \Delta \tilde{S}(t)$ starting with $w_{k}=1$ increases with the number of iterations, as shown mathematically, until it reaches a plateau. In all of the cases except for 7-D, it changes its sign from negative to positive values (see Fig. 3 in the Supplemental Material [23]), implying that complete segregation has been achieved in the training set. This segregation, except for 7-D, is also present in the test set (Fig. 4; see also Fig. 4 in the Supplemental Material [23]). In the 7-D case, $\Delta \Delta \tilde{S}(t)$ remains negative, indicating that such a high dimensional space is not fully reliable for segregation based on entropies. By choosing $\tilde{S}_{\mathrm{AML}}-\tilde{S}_{\text {normal }}$ so that $\sum_{k} w_{k}\left(t_{\max }\right)\left(\Delta S_{i=N_{\min }}^{k}+\Delta S_{i=A_{\max }}^{k}\right) / 2=0$, the approach correctly diagnoses the presence of AML in all of the cases of the test set from one to six dimensions (Fig. 4; see also Fig. 4 in the Supplemental Material [23]).

\section{DISCUSSION}

The use of physical approaches has been remarkably successful in the characterization of heterogeneous cell 
populations and their evolution in many complex biological scenarios [24-27] to the extent of making inroads in mainstream biomedical research $[28,29]$. One of the main problems posed by hematological cancers, such as AML, is the underlying heterogeneity resulting from diverse molecular changes in the cellular state, including several recurrent mutations and chromosome translocations $[10,17]$. This heterogeneity is responsible, to a large degree, for the observed different clinical courses and is characterized at the cellular level by changes in size and granularity and by the acquisition and loss of characteristic cell surface markers. Traditional approaches to diagnose AML seek to identify subpopulations of cells with these characteristic changes induced by the disease $[2,9]$.

The analysis presented shows that, despite the heterogeneity present, it is possible to define an average characterization of AML and normal cell populations on multidimensional morphological and molecular landscapes that can be used to accurately diagnose the presence of this type of cancer. This approach shares many similarities with traditional statistical thermodynamic methods that use entropy to collapse many nonmeasurable degrees of freedom within just a few key macroscopic or mesoscopic variables. In the case of cell populations, the quantities analogous to the macroscopic and mesoscopic variables are the combinations of attributes that define multidimensional morphological and molecular landscapes for each population type. This analogy establishes a straightforward link between statistical thermodynamics and biomedical applications and opens the door to the use of the sophisticated statistical physics methodology to tackle problems of direct medical importance.

\section{ACKNOWLEDGMENTS}

I thank Wade T. Rogers for providing the data for the DREAM6/FlowCAP2 Molecular Classification of Acute Myeloid Leukemia Challenge prior to publication and for stimulating discussions. This work was supported by the MINECO under Grant No. FIS2012-38105.

[1] L. W. Terstappen, S. Konemann, M. Safford, M. R. Loken, K. Zurlutter, T. Buchner, W. Hiddemann, and B. Wormann, Flow Cytometric Characterization of Acute Myeloid Leukemia. Part I. Significance of Light Scattering Properties, Leukemia 5, 315 (1991).

[2] L. W. Terstappen, M. Safford, S. Konemann, M. R. Loken, K. Zurlutter, T. Buchner, W. Hiddemann, and B. Wormann, Flow Cytometric Characterization of Acute Myeloid Leukemia. Part II. Phenotypic Heterogeneity at Diagnosis, Leukemia 6, 70 (1992).

[3] C. E. Pedreira, E. S. Costa, Q. Lecrevisse, J. J. van Dongen, and A. Orfao (EuroFlow Consortium), Overview of Clinical Flow Cytometry Data Analysis: Recent Advances and Future Challenges, Trends Biotechnol. 31, 415 (2013).
[4] A. Yen, Flow Cytometry: Advanced Research and Clinical Applications (CRC Press, Boca Raton, FL, 1989).

[5] D. F. Keren, C. A. Hanson, and P. E. Hurtubise, Flow Cytometry and Clinical Diagnosis (ASCP Press, Chicago, 1994).

[6] L. A. Herzenberg, J. Tung, W. A. Moore, L. A. Herzenberg, and D. R. Parks, Interpreting Flow Cytometry Data: A Guide for the Perplexed, Nat. Rev. Immunol. 7, 681 (2006).

[7] S.C. Bendall et al., Single-Cell Mass Cytometry of Differential Immune and Drug Responses across a Human Hematopoietic Continuum, Science 332, 687 (2011).

[8] W. T. Rogers and H. A. Holyst, FlowFP: A Bioconductor Package for Fingerprinting Flow Cytometric Data, Adv. Bioinformatics 2009, 193947 (2009).

[9] N. Aghaeepour, G. Finak, H. Hoos, T. R. Mosmann, R. Brinkman, R. Gottardo, and R. H. Scheuermann, Critical Assessment of Automated Flow Cytometry Data Analysis Techniques, Nat. Methods 10, 228 (2013).

[10] C. Ortolani, Flow Cytometry Of Hematological Malignancies (Wiley-Blackwell, Chichester, England, 2011).

[11] S. R. d. Groot and P. Mazur, Non-equilibrium Thermodynamics (North-Holland, Amsterdam, 1962).

[12] DREAM6/FlowCAP2 data sets, http://flowrepository.org/ id/FR-FCM-ZZYA.

[13] DREAM6/FlowCAP2 Molecular Classification of AML Challenge results, http://www.the-dream-project.org/result/ classification-aml.

[14] G. Stolovitzky, D. Monroe, and A. Califano, Dialogue on Reverse-Engineering Assessment and Methods: The DREAM of High-Throughput Pathway Inference, Ann. N.Y. Acad. Sci. 1115, 1 (2007).

[15] R. J. Prill, J. Saez-Rodriguez, L. G. Alexopoulos, P. K. Sorger, and G. Stolovitzky, Crowdsourcing Network Inference: The DREAM Predictive Signaling Network Challenge, Sci. Signal. (Online) 4, mr7 (2011).

[16] P. Meyer et al., Verification of Systems Biology Research in the Age of Collaborative Competition, Nat. Biotechnol. 29, 811 (2011).

[17] R. A. Weinberg, The Biology of Cancer (Garland Science, New York, 2007).

[18] D. Reguera, J. M. Rubi, and J. M. G. Vilar, The Mesoscopic Dynamics of Thermodynamic Systems, J. Phys. Chem. B 109, 21502 (2005).

[19] D. A. Beard and H. Qian, Chemical Biophysics: Quantitative Analysis of Cellular Systems (Cambridge University Press, Cambridge, England, 2008).

[20] S. Kullback and R. A. Leibler, On Information and Sufficiency, Ann. Math. Stat. 22, 79 (1951).

[21] R. C. Tolman, The Principles of Statistical Mechanics, The International Series of Monographs on Physics (Clarendon Press, Oxford, England, 1938).

[22] A. Einstein, Theorie der Opaleszenz von Homogenen Flüssigkeiten und Flüssigkeitsgemischen in der Nähe des Kritischen Zustandes, Ann. Phys. (Berlin) 338, 1275 (1910).

[23] See Supplemental Material at http://link.aps.org/ supplemental/10.1103/PhysRevX.4.021038 for details of the implementation of Team21's solution to the DREAM6/FlowCAP2 Molecular Classification of Acute 
Myeloid Leukemia Challenge, the source code of two python files, and four supplemental figures.

[24] E. Kussell and S. Leibler, Phenotypic Diversity, Population Growth, and Information in Fluctuating Environments, Science 309, 2075 (2005).

[25] D. Jordan, S. Kuehn, E. Katifori, and S. Leibler, Behavioral Diversity in Microbes and Low-Dimensional Phenotypic Spaces, Proc. Natl. Acad. Sci. U.S.A. 110, 14018 (2013).

[26] O. Feinerman, J. Veiga, J. R. Dorfman, R. N. Germain, and G. Altan-Bonnet, Variability and Robustness in T Cell
Activation from Regulated Heterogeneity in Protein Levels, Science 321, 1081 (2008).

[27] F. Michor, T. P. Hughes, Y. Iwasa, S. Branford, N. P. Shah, C. L. Sawyers, and M. A. Nowak, Dynamics of Chronic Myeloid Leukaemia, Nature (London) 435, 1267 (2005).

[28] D. B. Agus and F. Michor, The Sciences Converge to Fight Cancer, Nat. Phys. 8, 773 (2012).

[29] F. Michor, J. Liphardt, M. Ferrari, and J. Widom, What Does Physics Have to do with Cancer?, Nat. Rev. Cancer 11, 657 (2011). 